\title{
HOMOLOGICAL EMBEDDING PROPERTIES OF THE FIBERS OF A MAP AND THE DIMENSION OF ITS IMAGE
}

\author{
JOHN J. WALSH ${ }^{1}$
}

\begin{abstract}
A relationship is established between the homological codimension of the point inverses of a map and the dimension of its image. An infinite-dimensional version leads to the conclusion that the image of a proper map defined on Hilbert space cannot be countable dimensional. A finite-dimensional version yields: if $g$ : $M^{n} \rightarrow Y$ is a proper map, $M^{n}$ is a $G$-orientable $n$-manifold without boundary, and $\operatorname{dim} Y \leqslant k$, then there is a point $y \in Y$ and an integer $i \geqslant n-k$ such that $\check{H}^{i}\left(g^{-1}(y) ; G\right) \neq 0$.
\end{abstract}

Introduction. An argument based on a routine application of the Mayer-Vietoris sequence establishes a relationship between the homological codimension of the point inverses of a map and the dimension of its image. The motivating factor for extracting such a relationship was to establish that a proper map defined on Hilbert space cannot have an image that is countable dimensional. An analysis of this infinite-dimensional result exposed a parallel finite-dimensional version that extends a classical result for the class of dimension lowering maps defined on manifolds without boundary.

The main results are stated and proved in $\$ 2$. Listed below are four corollaries; their derivations appear in $\$ 3$.

Corollary 1. Let $g: l_{2} \rightarrow Y$ be a surjective proper map defined on a Hilbert space. Then $Y$ is not countable dimensional.

Properness is needed primarily to assure that each point inverse is a $Z$-set and, consequently, has infinite codimension (the salient feature needed in the proof). In general form, there is

COROLlary 2. Let $g: X \rightarrow Y$ be a surjective map from a nonempty ANR to a completely metrizable space such that each point inverse has infinite codimension in $X$. Then $Y$ is not countable dimensional.

Completeness plays an essential role even for $g$ a proper map. Points in the subspace $l_{2}^{f}=\left\{\left(x_{i}\right) \in l_{2}: x_{i}=0\right.$ for all but finitely many $\left.i\right\}$ are $Z$-sets in $l_{2}^{f}[4]$ and, therefore, the point inverses of the identity map of $l_{2}$ have infinite codimension but $l_{2}^{f}$ is countable dimensional.

Received by the editors July 10, 1981.

1980 Mathematics Subject Classification. Primary 54F45, 58B05; Secondary 54C05, 55M10, 55N05.

Key words and phrases. Z-set, infinite codimension, countable dimensional, homological embedding properties, dimension, Hilbert space, Hilbert cube.

' Research supported in part by NSF Grant. 
It is a classical result that for a proper map $g: X \rightarrow Y$ there is a point $y \in Y$ such that

$$
\operatorname{dim} g^{-1}(y) \geqslant \operatorname{dim} X-\operatorname{dim} Y
$$

(see [7, p. 91]). For proper maps defined on manifolds without boundary, the preceding inequality is detected by cohomology.

COROLlARY 3. Let $\mathrm{g}: \boldsymbol{M}^{n} \rightarrow Y$ be a proper map defined on a nonempty G-orientable $n$-manifold without boundary and suppose that $\operatorname{dim} Y \leqslant k$. Then there is a point $y \in Y$ such that $\check{H}^{i}\left(f^{-1}(y) ; G\right) \neq 0$ for some $i \geqslant n-k$.

By considering the restriction of $f$ to $g^{-1}(U)$ for open subsets $U$ of $Y$, it follows that the points $y \in Y$ satisfying the conclusion of the previous corollary form a dense subset of $f(Y)$.

A more discerning feature of the finite-dimensional version of the main result is illustrated by considering maps $g: S^{k} \times S^{k} \rightarrow I$ from the product of two $k$-spheres onto the interval $I=[0,1]$. While the conclusion of Corollary 3 follows immediately since the nonendpoints separate $I$, the main result also yields

Corollary 4. Let g: $S^{k} \times S^{k} \rightarrow I$ be a surjective map. Then there is a point $t \in I$ such that either $\check{H}^{k}\left(g^{-1}(t) ; \mathbf{Z}\right) \neq 0$ or $\check{H}^{k-1}\left(g^{-1}(t) ; \mathbf{Z}\right) \neq 0$.

The next example illustrates that there only need be a single point $t$ satisfying the conclusion of this last corollary for $k \geqslant 3$. The complement in $S^{k} \times S^{k}$ of the wedge $S^{k} \times\{\mathrm{pt}\} \cup\{\mathrm{pt}\} \times S^{k}$ is an open $2 k$-cell and, therefore, there is an easily defined map $g: S^{k} \times S^{k} \rightarrow I$ such that $g^{-1}(0)=$ point, $g^{-1}(1)=S^{k} \times\{\mathrm{pt}\} \cup\{\mathrm{pt}\} \times S^{k}$, and $g^{-1}(t)=S^{2 k-1}$ for $0<t<1$.

1. Preliminaries. It is convenient to require that spaces be separable and metric. In general, the term map (or mapping) is used to specify a continuous function. A map $f: X \rightarrow Y$ is proper provided $f^{-1}(C)$ is compact for each compact subset $C$ of $Y$.

The homology theory used is singular theory with coefficients in an abelian group and the cohomology theory used is Čech theory with coefficients in an abelian group. A compact carrier for a singular homology element $z \in H_{q}(U, V)$ is a pair of compacta $(P, \partial P) \subset(U, V)$ for which $z \in \operatorname{Im}\left\{i_{*}: H_{q}(P, \partial P) \rightarrow H_{q}(U, V)\right\}$. A natural source for carriers is the union of images of the maps in a singular chain representing $z$.

A closed subset $A$ of an ANR (absolute neighborhood retract) $X$ is called a $Z$-set provided the homotopy groups $\pi_{*}(U, U-A)$ are trivial for each open subset $U$ of $X$. The closed set $A$ is said to have infinite codimension provided the homology groups $H_{*}(U, U-A ; \mathbf{Z})$ are trivial for each open subset $U$ of $X$. For coefficient group $G$ other than $\mathbf{Z}$, we shall say $A$ has infinite codimension with respect to the coefficient group $G$. The reader is referred to [3] and [6] for further details on $Z$-sets and infinite codimension, respectively.

The covering dimension of a space $X$ is denoted $\operatorname{dim} X$. A space is countable dimensional provided it is the countable union of zero-dimensional spaces. Following [7, p. 50], a space $X$ has small transfinite inductive dimension, written ind $X \leqslant \alpha$, 
provided $\alpha$ is an ordinal and, for each $x \in X$ and $\varepsilon>0$, there is a neighborhood $U$ of $x$ having diameter $<\varepsilon$ such that ind(Fr $U)<\alpha$.

Amongst completely metrizable spaces, the countable-dimensional ones are precisely those that possess small transfinite inductive dimension [7, p. 51]. Consequently, neither Hilbert spaces nor the Hilbert cube possess such dimension nor does the countable-dimensional subspace $l_{2}=\left\{\left(x_{i}\right) \in l_{2}: x_{i}=0\right.$ for all but finitely many $i$ ]. The reader is referred to [7] for further information on dimension theory.

2. Statements and proofs of main theorems. The main result is recorded in two forms. An essentially infinite-dimensional version that extends results in $[6, \S 2]$ and a finite-dimensional version that is a "parametrized" form of a known result that is recovered in Theorem B by insisting that $K=Y$ and $f$ be the identity (see [5, Appendix]). In all cases, the critical part of the proof is a routine Mayer-Vietoris argument.

THEOREM A. Let $Y$ be a space having small transfinite inductive dimension, let $K$ be a closed subset of an $A N R X$, and let $f: K \rightarrow Y$ be a map with each point-inverse $f^{-1}(y)$ having infinite codimension in $X$ with respect to a coefficient group $G$. Then $K$ has infinite codimension in $X$ with respect to the coefficient group $G$.

TheOREM B. Let $Y$ be a finite-dimensional space, let $K$ be a closed subset of an ANR $X$, let $f: K \rightarrow Y$ be a map, and let $q$ be an integer such that $H_{i}\left(X, X-f^{-1}(y) ; G\right)=0$ for each $y \in Y$ and $q \leqslant i \leqslant q+\operatorname{dim} Y$. Then $H_{q}(X, X-K ; G)=0$.

Both proofs proceed by induction on the dimension of $Y$, the induction being transfinite in one case. The slightly more difficult proof of Theorem $B$ is presented next while the easy task of extracting a proof of Theorem $A$ is left to the reader. The coefficient group $G$ is suppressed.

The conclusion is trivially satisfied for $\operatorname{dim} Y=-1$ as $K$ must be empty. Inductively, we assume not only that $H_{q}\left(X, X-f^{-1}(L)\right)=0$ for each closed subset $L \subset Y$ with $\operatorname{dim} L<\operatorname{dim} Y$, but also that $H_{q+1}\left(X, X-f^{-1}(L)\right)=0$, since $H_{i}\left(X, X-f^{-1}(y)\right)=0$ for each $y \in L$ and $q \leqslant i \leqslant q+1+\operatorname{dim} L$.

The remainder of the argument uses the observation that, for a pair of closed subsets $A$ and $B$ of $X$, the Mayer-Vietoris sequence for "the excisive couple of pairs" $\{(X, X-A),(X, X-B)\}$ yields an inclusion induced isomorphism

$$
H_{q}(X, X-(A \cup B)) \rightarrow H_{q}(X, X-A) \oplus H_{q}(X, X-B)
$$

whenever the adjacent terms $H_{q}(X, X-(A \cap B))$ and $H_{q+1}(X, X-(A \cap B))$ are trivial.

Fix $z \in H_{q}(X, X-K)$ and choose a compact carrier $(P, \partial P)$ for $z$. Specify a neighborhood $V_{y}$ of each $y \in f(P \cap K)$ such that the image of $z$ in $H_{q}\left(X, X-f^{-1}\left(V_{y}\right)\right)$ is trivial; this is possible since by hypothesis $H_{q}\left(X, X-f^{-1}(y)\right)$ $=0$ and, therefore, $z$ has a compact carrier missing $f^{-1}(y)$. Refine the cover $\left\{V_{y}\right\}$ of $f(P \cap K)$ by a cover $\left\{R_{i}: i=1,2, \ldots, k\right\}$ of $f(P)$ by closed sets such that the interior of $\cup_{i=1}^{k} R_{i}$ contains $f(P \cap K)$ and the frontier of each $R_{i}$ has dimension strictly less than the dimension of $Y$. 
Define sets $A_{j}=C l\left(Y-\cup_{i=j+1}^{k} R_{i}\right)$ where $j=0,1, \ldots, k$. Since $A_{0}$ does not intersect $f(P \cap K)$, the image of $z$ in $H_{q}\left(X, X-f^{-1}\left(A_{0}\right)\right)$ is trivial. Inductively, for $j=1,2, \ldots, k$, we assume that the image of $z$ in $H_{q}\left(X, X-f^{-1}\left(A_{j-1}\right)\right)$ is trivial and, by choice, its image is trivial in $H_{q}\left(X, X-f^{-1}\left(R_{j}\right)\right)$. Since $f^{-1}\left(A_{j-1}\right) \cap f^{-1}\left(R_{j}\right)=$ $f^{-1}\left(A_{j-1} \cap R_{j}\right)$ and $\operatorname{dim} A_{j-1} \cap R_{j}<\operatorname{dim} Y$ as $A_{j-1} \cap R_{j} \subset \operatorname{Fr} R_{j}$, we have inductively that $H_{e}\left(X, X-\left(f^{-1}\left(A_{j-1}\right) \cap f^{-1}\left(R_{j}\right)\right)\right)$ is trivial for $e=q, q+1$ and the Mayer-Vietoris argument given earlier yields an isomorphism

$$
\begin{aligned}
H_{q}\left(X, X-\left(f^{-1}\left(A_{j-1}\right)\right.\right. & \left.\left.\cup f^{-1}\left(R_{j}\right)\right)\right) \\
& \rightarrow H_{q}\left(X, X-f^{-1}\left(A_{j-1}\right)\right) \oplus H_{q}\left(X, X-f^{-1}\left(R_{j}\right)\right) .
\end{aligned}
$$

We conclude that the image of $z$ in $H_{q}\left(X, X-f^{-1}\left(A_{j}\right)\right)$ is trivial. Finally, when $j=k$ we see that $z$ itself is trivial.

\section{Proofs of corollaries.}

Proofs of Corollaries 1 AND 2. Since the image of a proper map defined on a complete metric space is completely metrizable [9], the first corollary follows from the second as compact subsets of $l_{2}$ are $Z$-sets [2]. Corollary 2 is proved by way of contradiction. If $Y$ were countable dimensional, then $Y$ would have small transfinite inductive dimension [7, p. 51] and Theorem A would apply with $G=\mathbf{Z}$ and $K=X$ and would yield that $X$ has infinite codimension in $X$. This is not possible since $H_{0}(X, X-X ; \mathbf{Z})=H_{0}(X ; \mathbf{Z}) \neq 0$.

Proof of Corollary 3. There is a point $y \in Y$ and an integer $0<i<\operatorname{dim} Y$ such that $H_{i}\left(M, M-f^{-1}(y) ; G\right) \neq 0$; otherwise, Theorem B would apply with $K=M$ and $q=0$ and would contradict that $H_{0}(M, M-M ; G)=H_{0}(M ; G) \neq 0$. Alexander duality [8, p. 296] yields that $\check{H}^{n-i}\left(f^{-1}(y) ; G\right) \neq 0$ and it is transparent that $n-i \geqslant n-\operatorname{dim} Y \geqslant n-k$.

Proof of Corollary 4. In order to avoid being able to use Theorem B with $K=X, G=\mathbf{Z}$, and $q=k$ and contradicting that $H_{k}\left(S^{k} \times S^{k} ; \mathbf{Z}\right) \neq 0$, there must be a point $t \in I$ such that $H_{e}\left(S^{k} \times S^{k}, S^{k} \times S^{k}-f^{-1}(y) ; Z\right) \neq 0$ for one of $e=k$, $k+1$. Alexander duality yields that either $\check{H}^{k}\left(f^{-1}(y) ; \mathbf{Z}\right) \neq 0$ or $\check{H}^{k-1}\left(f^{-1}(y) ; \mathbf{Z}\right)$ $\neq 0$.

\section{REFERENCES}

1. F. D. Ancel, On ANR's and cell-like maps, Seminar Notes, University of Oklahoma, 1980.

2. R. D. Anderson, Topological properties of the Hilbert cube and the infinite product of open intervals, Trans. Amer. Math. Soc. 126 (1967), 200-216.

3. T. A. Chapman, Lectures on Hilbert cube manifolds, CBMS Regional Conf. Ser. in Math., no. 28, Amer. Math. Soc., Providence, R.I., 1976.

4. , Dense sigma-compact subsets of infinite-dimensional manifolds, Trans. Amer. Math. Soc. 154 (1971), 399-426.

5. R. J. Daverman, Detecting the disjoint disks property, Pacific J. Math. 93 (1981), 277-298.

6. R. J. Daverman and J. J. Walsh, Čech homology characterizations of infinite dimensional manifolds, Amer. J. Math. 103 (1981), 411-435.

7. W. Hurewicz and H. Wallmann, Dimension theory, Princeton Univ. Press, Princeton, N.J., 1948.

8. E. H. Spanier, Algebraic topology, McGraw-Hill, New York, 1966.

9. I. A. Vainsteìn, On closed mappings of metric spaces, Dokl. Akad. Nauk SSSR 57 (1947), 319-321.

Department of Mathematics, University of Tennessee, Knoxville, Tennessee 37916 